\title{
Urinary tract infection in a clinic for sexually transmitted diseases
}

\author{
M. G. MEAD AND R. N. GRÜNEBERG \\ From the Departments of Microbiology and Genitourinary Medicine, \\ University College Hospital, London WC1
}

SUMMARY A study of the prevalence of urinary tract infection (UTI) in 430 women attending a clinic for sexually transmitted diseases and its relationship to sexual activity is presented. UTI, usually asymptomatic, was found to be slightly more common in women attending the clinic than in the general population, its prevalence being $4.9 \%$. UTI was more commonly found in patients who gave a history of recent sexual intercourse, which suggests that recent coitus was a factor in the development of significant bacteriuria. The likelihood of finding significant bacteriuria was not related to the number of sexual partners in the previous year. Trichomoniasis was more common in the small group of patients with UTI than in other women. The most common causative organism of UTI was Escherichia coli, and the isolates were usually sensitive to ampicillin, sulphonamides, trimethoprim, and nitrofurantoin.

\section{Introduction}

Urinary tract infection (UTI) is a very common problem in clinical practice, yet it is still little understood. It is diagnosed by the finding of significant bacteriuria, a pure growth of more than 100000 organisms $/ \mathrm{ml}$ of urine from two consecutive specimens. The terms 'urinary infection' and 'significant bacteriuria' are used synonymously here, as in most recent publications. Significant bacteriuria may or may not be associated with symptoms such as frequency and dysuria. Asymptomatic bacteriuria is important in pregnancy, when it may be associated with pyelonephritis in the later months (Kass, 1960).

The prevalence of significant bacteriuria varies with both age and sex. It is rare in adult males. In females it varies from about $1-2 \%$ in five-year-old schoolgirls (Savage and Wilson, 1973) to $3-5 \%$ in young, sexually active women The greater prevalence of UTI in women during the child-bearing years than before the menarche has led to the suggestion that coitus may play a role in its pathogenesis (Grüneberg, 1970). Support for this suggestion has come from various studies. Kunin (1970), in a

Address for reprints: M. G. Mead, Department of Microbiology, University College Hospital, Gower Street, London WC1E 6AU

Received for publication 15 December 1977 long-term study of recurrent bacteriuria in schoolgirls, found that sexual intercourse was related to the acquisition of bacteriuria and of symptomatic infections. Sexual intercourse is indeed often recognised by patients as a precipitating factor in the development of a urinary tract infection (Grüneberg, 1970).

Kunin and McCormack (1968) compared the prevalence of significant bacteriuria in 2882 white nuns with that in $\mathbf{2 3 0 2}$ white, working women (most of whom were married). They found that in the age group 15-34 years significant bacteriuria was $12 \cdot 8$ times more common in working women than in nuns, whereas in the age group 55 and over it was only 1.4 times as common. Only two $(0.3 \%)$ of 698 white nuns aged 25-34 years had significant bacteriuria. In view of the possible association with sexual intercourse it would be of interest to know its prevalence in a clinic for sexually transmitted diseases (STD). The present study had three aims: firstly, to determine the prevalence of urinary tract infection in female patients attending a clinic for STD; secondly, to investigate the relationship, if any, of sexual activity to the finding of significant bacteriuria; and, thirdly, to ascertain whether there was a relationship between the presence of urinary tract infection and the presence of gonorrhoea, trichomoniasis, or candidosis. 


\section{Materials and methods}

STUDY POPULATION

The series comprised 430 consecutive, unselected female patients attending the Department of Genitourinary Medicine, University College Hospital. Fewer than $5 \%$ of them had been seen before in the clinic and none within the previous six months. Each patient was asked her age, the number of sexual partners she had had in the last year, the date of the last sexual intercourse, and whether she had taken antibiotics during the last three months.

\section{CLINICAL PROCEDURES}

Patients were examined in the lithotomy position and specimens taken from the vagina, cervix, and urethra for microscopical examination and culture for Neisseria gonorrhoeae, Trichomonas vaginalis, and Candida albicans. After examination each patient provided a midstream urine specimen (MSU), which was immediately stored at $4^{\circ} \mathrm{C}$. Each patient with UTI was treated with an appropriate antibiotic and followed up to establish that the infection had been eradicated.

\section{LABORATORY PROCEDURES}

Urethral and cervical smears were Gram-stained for intracellular Gram-negative diplococci; cultures for $N$. gonorrhoeae were inoculated on to VCNT medium (Phillips et al., 1972). Vaginal specimens were suspended in saline and examined microscopically for trichomonads and for yeast cells and hyphae. Culture for C. albicans was on Sabouraud's medium. $T$. vaginalis was not cultured.

The MSU specimen was screened for bacteriuria within six hours by the method of Leigh and Williams (1964) on cystine lactose electrolytedeficient (CLED) medium. Each specimen was plated in duplicate. After overnight incubation those with over 30 colonies of pure growth per inoculum area were examined by routine bacteriological methods to confirm the presence of bacteriuria greater than $100000 / \mathrm{ml}$ and to identify and determine the sensitivity of the bacterium. If a positive result was obtained a second specimen was requested at the patient's follow-up visit a few days later and was treated in the same way.

\section{Results}

The mean age of the 430 patients was 25.4 years and the median age was 24 years; over $75 \%$ of the patients were aged between 18 and 30 years.

The mean number of sexual partners for each of the patients in the previous year was two. Twelve patients said they had had no sexual partners in the previous year, 212 had had one, 116 had had two, 40 had had three, 24 had had four, and 26 patients had had five or more.

When the MSUs from the 430 patients were screened, 23 were found to yield a pure growth of greater than 100000 bacteria/ml on the first occasion; of these patients, 10 again had a positive result on a second consecutive specimen a few days later while two had a negative result on the second occasion and were excluded from the study. The remaining 11 patients had a positive culture on the first occasion but did not have a further MSU examination on a follow-up visit; of these, six failed to attend for follow-up, and five had been treated with antibiotics at the first visit (two because of dysuria and frequency). All these 11 patients have been included in the study as having UTI despite the degree of uncertainty regarding diagnosis, as a single specimen is only $80 \%$ reliable as an indicator of infection (Kass, 1956). Thus, 21 out of 430 women had UTI, giving a prevalence of $4.9 \%-\mathrm{a}$ similar result to that found in patients attending antenatal clinics (Grüneberg et al., 1969).

The relationship of the number of sexual partners to the diagnosis of UTI, gonorrhoea, candidosis, and trichomoniasis is shown in Table 1. Gonorrhoea was present in $30(7 \%)$ of the 430 patients, candidosis was present in $99(23.0 \%)$, and $t_{1}$ ichomoniasis was present in $35(8.1 \%)$. Of the 30 patients with gonorrhoea, seven had an associated trichomoniasis but none had UTI. Of the 99 patients with candidosis, five $(5.1 \%)$ had UTI; whereas of the 35 patients with trichomoniasis, three $(8.6 \%)$ had UTI. The overall prevalence of UTI in the 430 patients was $4.9 \%$; thus UTI was more common in those patients with trichomoniasis.

The interval between the last coitus and the attendance of the patient at the clinic is shown in Table 2. Four of the patients had urinary symptoms,

Table 1 Diagnosis in relation to number of sexual partners in previous year

\begin{tabular}{|c|c|c|c|c|c|c|c|c|c|c|c|}
\hline \multirow{3}{*}{$\begin{array}{l}\text { No. of sexual } \\
\text { partners }\end{array}$} & \multirow{3}{*}{$\begin{array}{l}\text { No. of } \\
\text { patients }\end{array}$} & \multirow{2}{*}{\multicolumn{2}{|c|}{$U T I$}} & \multicolumn{8}{|c|}{ Diagnosis* } \\
\hline & & & & \multicolumn{2}{|c|}{ Gonorrhoea } & \multicolumn{2}{|c|}{ Candidosis } & \multicolumn{2}{|c|}{ Trichomoniasis } & \multicolumn{2}{|c|}{ None of these } \\
\hline & & No. & $\%$ & No. & $\%$ & No. & $\%$ & No. & $\%$ & No. & $\%$ \\
\hline $0-2$ & 340 & 16 & $4 \cdot 7$ & 18 & $5 \cdot 3$ & 83 & $24 \cdot 4$ & 26 & $7 \cdot 6$ & 220 & $64 \cdot 7$ \\
\hline 3 or more & 90 & 5 & $5 \cdot 6$ & 12 & $13 \cdot 3$ & 16 & $17 \cdot 8$ & 9 & $10 \cdot 0$ & 57 & $63 \cdot 3$ \\
\hline Total & 430 & 21 & 4.9 & 30 & $7 \cdot 0$ & 99 & $23 \cdot 0$ & 35 & $8 \cdot 1$ & 277 & $64 \cdot 4$ \\
\hline
\end{tabular}

*Patients with more than one infection appear under each diagnosis 
Table 2 Interval between most recent coitus and attendance at the clinic

\begin{tabular}{lclc}
\hline Interval (days) & Patients & $U T I$ \\
\cline { 3 - 4 } & & No. & $\%$ \\
1 or less & 45 & $7^{*}$ & $15 \cdot 6$ \\
$2-4$ & 97 & $4^{*}$ & $4 \cdot 1$ \\
$5-14$ & 148 & 5 & $3 \cdot 4$ \\
$15-30$ & 68 & 3 & $4 \cdot 4$ \\
30 & 58 & 2 & $3 \cdot 4$ \\
\hline
\end{tabular}

*Two with urinary symptoms

and, of those, two presented within one day, one within two days, and one within three days of the most recent coitus. Fourteen patients were not asked the date of their last coitus.

Details of patients with UTI and the organisms isolated are shown in Table 3. In all but four instances the organism causing the infection was Escherichia coli; in the other instances the organisms were Klebsiella species (2), Staphylococcus albus, and a Micrococcus species. Sixteen of the 21 organisms were sensitive to ampicillin, sulphonamides, trimethoprim, and nitrofurantoin. Four of the organisms were resistent to sulphonamides. Only four of the 21 patients had urinary symptoms; most presented with vaginal discharge or because the sexual contact had symptoms.

\section{Discussion}

This study indicates that $4.9 \%$ of female patients attending a clinic for STD, mostly for the first time, had significant bacteriuria. This is a slightly higher percentage than would be expected in a population of healthy, young women. Asscher et al. (1969), for example, found significant bacteriuria in $3 \%$ of 3578 healthy women aged 20-65. Grüneberg et al. (1969) found significant bacteriuria in $4.4 \%$ of 8907 women attending an antenatal clinic. In this study the prevalence of UTI almost equalled that of gonorrhoea $(7 \%)$. This raises the question of the value of screening. There is no doubt that UTI can cause problems in pregnancy. Kincaid-Smith and Bullen (1965), for example, found that fetal loss and prematurity (fetal weight less than 2500 grams) were significantly increased in pregnant women with bacteriuria compared with women without. There is also a danger of pyelonephritis in pregnancy (Kass, 1960). There have been many reports that women with bacteriuria often have radiological evidence of renal abnormality. The investigation and treatment of women with bacteriuria detected by screening might help to reduce morbidity in a subsequent pregnancy. The women attending a STD clinic are, by virtue of their age and sexual activity, very likely to become pregnant; in fact, pregnancy is often first diagnosed at the clinic. All new patients have a routine urine examination for protein and sugar, and the screening of urine samples for the presence of significant bacteriuria is a quick and simple procedure (Leigh and Williams, 1964). Reliance on symptoms is insufficient; only four of the 21 women with positive cultures had urinary tract symptoms. However, treatment of UTI is often followed by reinfection, and some workers are sceptical of the value of screening for bacteriuria in women who are not pregnant (Asscher et al., 1969).

Table 3 Symptoms, urinary pathogens, and other diagnoses in patients with UTI

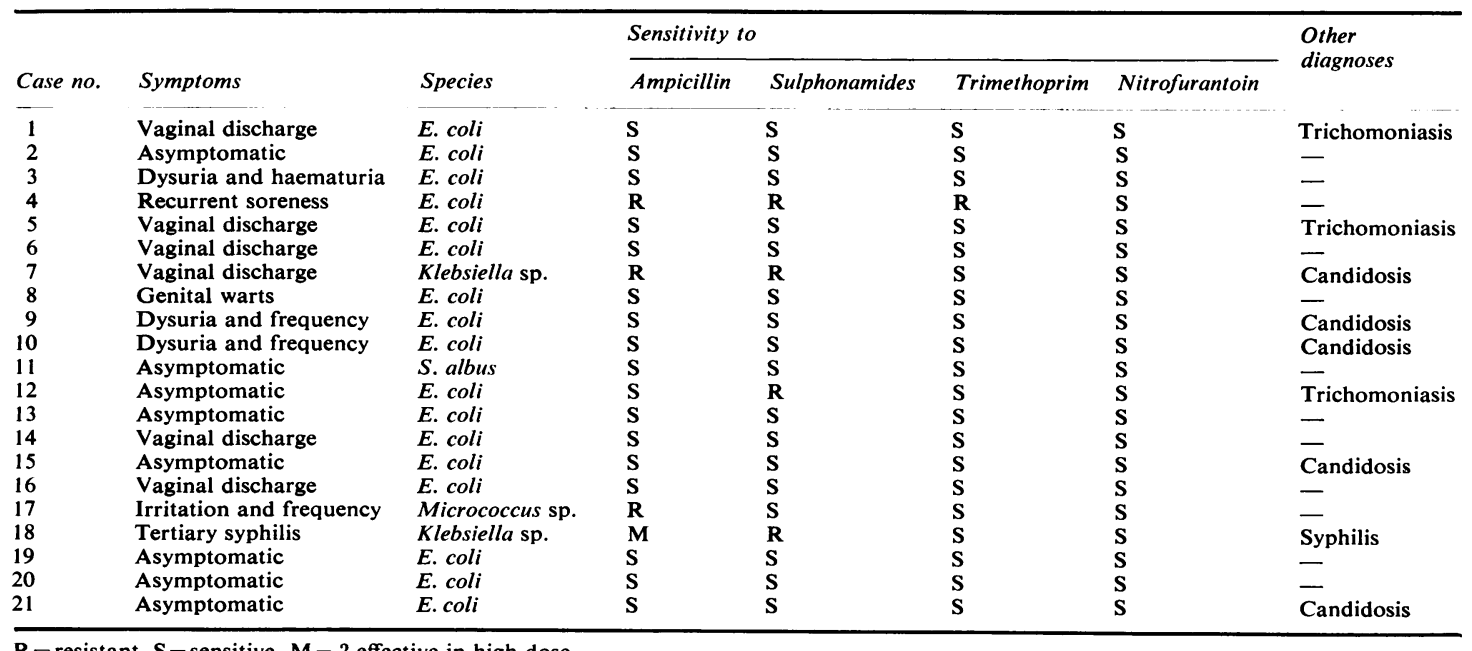


In this study we have shown (Table 2) that women with UTI are more likely to have had coitus during the previous 24 hours than are women without UTI. The explanation for this could be either that sexual intercourse causes a short-lasting bacteriuria which is then detected on screening or that women with UTI have coitus more frequently than uninfected women. In either case, coitus seems in some way to be a precipitating factor in the development of most episodes of UTI occurring in young women. This suggestion is often made but is rarely supported by objective data.

We have been unable to demonstrate in this study any relationship between the number of sexual partners in the previous year and the chance of a woman having UTI. There was, however, a greater prevalence of gonorrhoea and trichomoniasis in the more promiscuous group of women. These findings suggest that UTI may be related more to the frequency of coitus than to the number of sexual partners and is therefore probably not a sexually transmitted disease.

We would like to thank the staff of the Departments of Genitourinary Medicine and Microbiology, University College Hospital, for their help in this work and to Dr J. D. Oriel for allowing us to study patients under his care.
References

Asscher, A. W., Sussman, M., Waters, W. E., Evans, J. A. S., Campbell, H., Evans, K. T., and Williams, J. E. (1969). The clinical significance of asymptomatic bacteriuria in the nonpregnant woman. Journal of Infectious Diseases, 120, 17-21.

Grüneberg, R. N. (1970). Urinary tract infection in the female: a sexually induced disease? Technical Venereology Bulletin, 1, 9-11.

Grüneberg, R. N., Leigh, D. A., and Brumfitt, W. (1969). Relationship of bacteriuria in pregnancy to acute pyelonephritis, prematurity, and fetal mortality. Lancet, 2, 1-3.

Kass, E. H. (1956). Asymptomatic infection of the urinary tract. Transactions of the Association of American Physicians, 69, 56.

Kass, E. H. (1960). Bacteriuria and pyelonephritis of pregnancy. Archives of Internal Medicine, 105, 194-198.

Kincaid-Smith, P., and Bullen, M. (1965). Bacteriuria in pregnancy. Lancet, 1, 395-399.

Kunin, C. M. (1970). The natural history of recurrent bacteriuria in schoolgirls. New England Journal of Medicine, 282, 1443-1447.

Kunin, C. M., and McCormack, R. C. (1968). An epidemiologic study of bacteriuria and blood pressure among nuns and working women. New England Journal of Medicine, 278, 635-642.

Leigh, D. A., and Williams, J. D. (1964). Method for the detection of significant bacteriuria in large groups of patients. Journal of Clinical Pathology, 17, 498-503.

Phillips, I., Humphrey, D., Middleton, A., and Nicol, C. S. (1972). Diagnosis of gonorrhoea by culture on a selective medium containing vancomycin, colistin, nystatin, and trimethoprim (VCNT). A comparison with Gram-staining and immunofluorescence. British Journal of Venereal Diseases, 48, 287-292.

Savage, D. C. L., and Wilson, M. I. (1973). Covert bacteriuria of childhood. In L'rinary Tract Infection, pp. 39-47. Edited by W. Brumfitt and A. W. Asscher. Oxford University Press: London. 